\title{
Three Consecutive Monthly Intravitreal Ranibizumab for Choroidal Neovascularization in Central Serous Choriorethınopathy: A Case Report
}

\author{
Kazim Erol $^{1}$, Esin Sogutlu Sari ${ }^{2 *}$, Arif Koytak ${ }^{3}$, A. Karaçor ${ }^{1}$, D. T. Çoban ${ }^{1}$, M. Bulut ${ }^{1}$ \\ ${ }^{1}$ Ophthalmology Department, Antalya Training and Research Hospital, Antalya, Turkey; ${ }^{2}$ Ophthalmology Department, Kartal Training \\ and Research Hospital, Istanbul, Turkey; ${ }^{3}$ Ophthalmology Department, Bezmialem Vakif University, Istanbul, Turkey. \\ Email: *dresinsogutlu@gmail.com
}

Received March 19 $9^{\text {th }}, 2012$; revised April 25 $5^{\text {th }}, 2012$; accepted May $8^{\text {th }}, 2012$

\begin{abstract}
Purpose: The authors report the result of three consecutive monthly intravitreal ranibizumab injection for choroidal neovascularization (CNV) after bevacizumab injection for chronic central serous rethinopathy (CSR). Methods: A 48year-old man with chronic CSR was treated with intravitreal single dose $2.5 \mathrm{mg}$ bevacizumab. One year after CNV was occurred, and three consecutive monthly intravitreal ranibizumab injections were performed. Results: Four weeks later the first ranibizumab dose, best corrected visual acuity was improved 20/80 to 20/20 and remained stable within one year. Conclusion: Repeat intravitreal ranibizumab injection in CNV after bevacizumab injection for chronic CSR appeared to be an effective treatment option.
\end{abstract}

Keywords: Central Serous Choriorethinopathy; Choroidal Neovascularization; Ranibizumab

\section{Introduction}

Central serous chorioretinopathy (CSR) is common diseases of the posterior segment of the eye characterized by serous detachment of the neurosensory retina in the macula secondary to an idiopathic leakage in the outer blood-retinal barier at the retinal pigment epithelium (RPE). Although visual distortions are usually mild and spontaneous recovery occurs within a few months, some patients with CSR have a poor visual acuity due to retinal pigment epithelium atrophy, persistant or recurrent pigment epithelial detachment, subretinal fluid and choroidal neovascularization (CNV) [1]. CNV secondary to CSR is an uncommon relation which has been also noted to complicate laser photocoagulation treatment due to the puncture of Bruch's membrane by laser burns and photodynamic theraphy due to the RPE alterations and induces the release of vascular endothelial growth factor (VEGF) [2].

Different treatment options including photodynamic theraphy with vertaporfin, laser photocoagulation, vitroretinal submacular surgery and intravitreal anti VEGF agents (bevacizumab or ranibizumab) have been reported for the chronic and recurrent CSR with or without CNV. [2-5] We report the results of three consecutive monthly

${ }^{*}$ Corresponding author. intravitreal ranibizumab injection for $\mathrm{CNV}$ after bevacizumab injection for chronic CSR. To our knowledge there have been no previously reported cases of CNV after bevacizumab for the management of CSR.

\section{Case Report}

A 48-year-old man with a history of chronic ulcerative colitis had reported a 8-month history of blurry vision in his right eye and he had received prior medical treatment with acetazolamid. Metamorphopsia was noted and visual acuity was 20/40. RPE "sawtooth appearence" was present on optical coherence tomography (OCT) suggesting a chronic CSR (Figure 1). Treatment options were discussed and intravitreal bevacizumab $(2.5 \mathrm{mg})$ injection was performed. Four weeks later best corrected visual acuity was improved to 20/20 and OCT revealed complete resolution of neurosensory serous detachment (Figure 2).

One year after the bevacizumab injection, the patient complained of decreased vision in his right eye for the past 2 weeks. Best corrected visual acuity was 20/80 and metamorphopsia was again noted. OCT imaging showed CNV with subretinal fluid (Figure 3). Treatment options were discussed three consecutive monthly intravitreal ranibizumab injection $(0.5 \mathrm{mg})$ were performed. Four weeks later the last dose best corrected visual acuity was 
improved to 20/20 and OCT demonstretad the complete resolution of choroidal neovascular membrane and subretinal fluid (Figure 4). Follow-up examination at one year after the last ranibizumab injection vision remained stable on $20 / 20$.
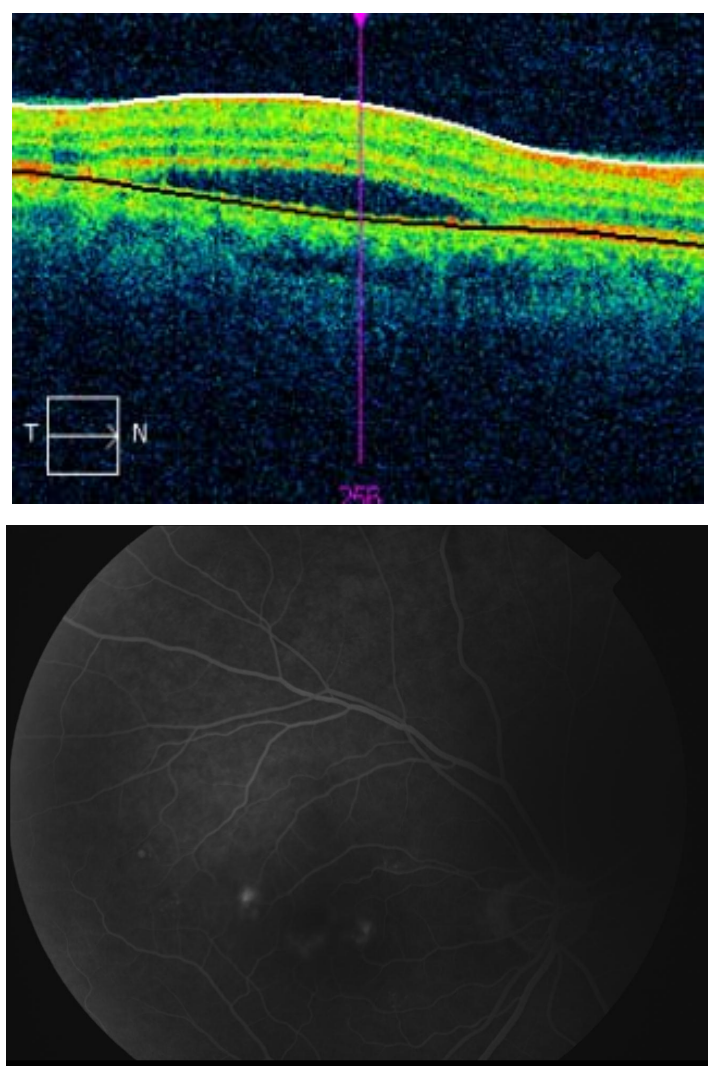

Figure 1. Baseline optical cohorence tomography (OCT) showed serous detachment of the neurosensory retina with central macular thickness of $389 \mu \mathrm{m}$ and "sawtooth appearence" of the retina pigment epithelium (RPE). Baseline flourescein angiography (FA) showed RPE "window defect" with focal leakage of flourescein.
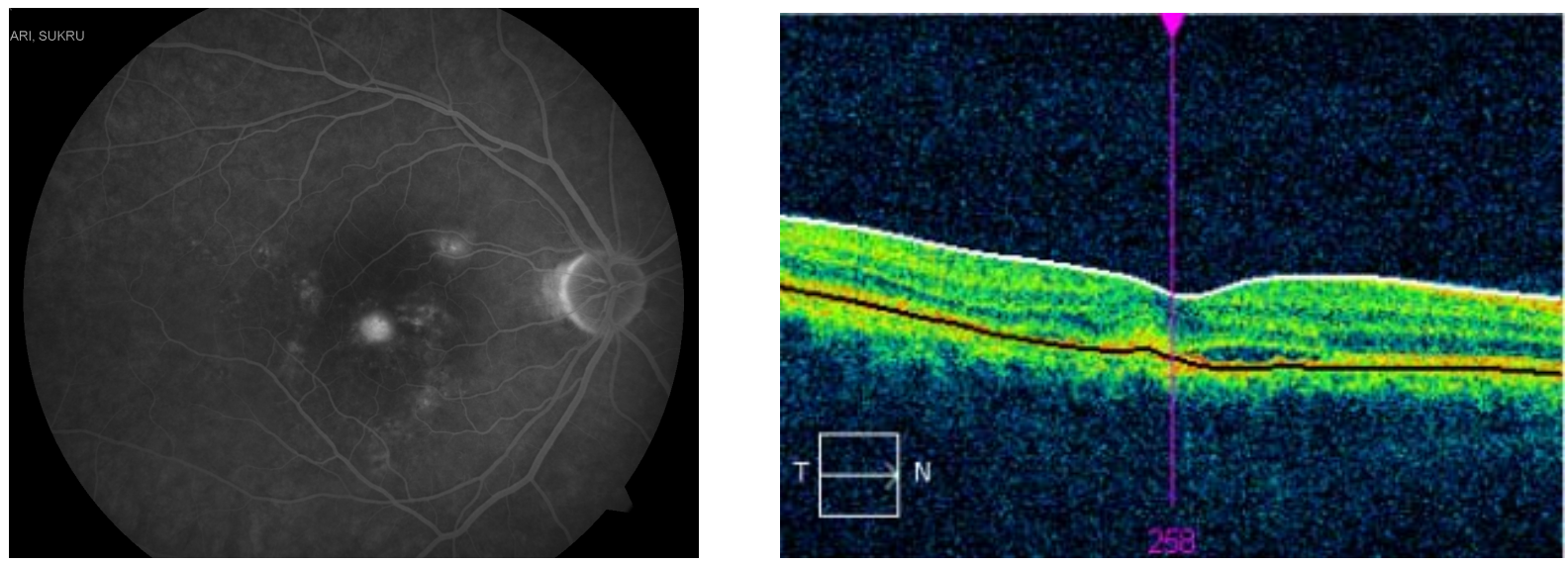

Figure 3. Flourescein angiography (late frames) and optical coherence tomography demonstrating a subfoveal classic choroidal neovascular membrane with subretinal fluid. 

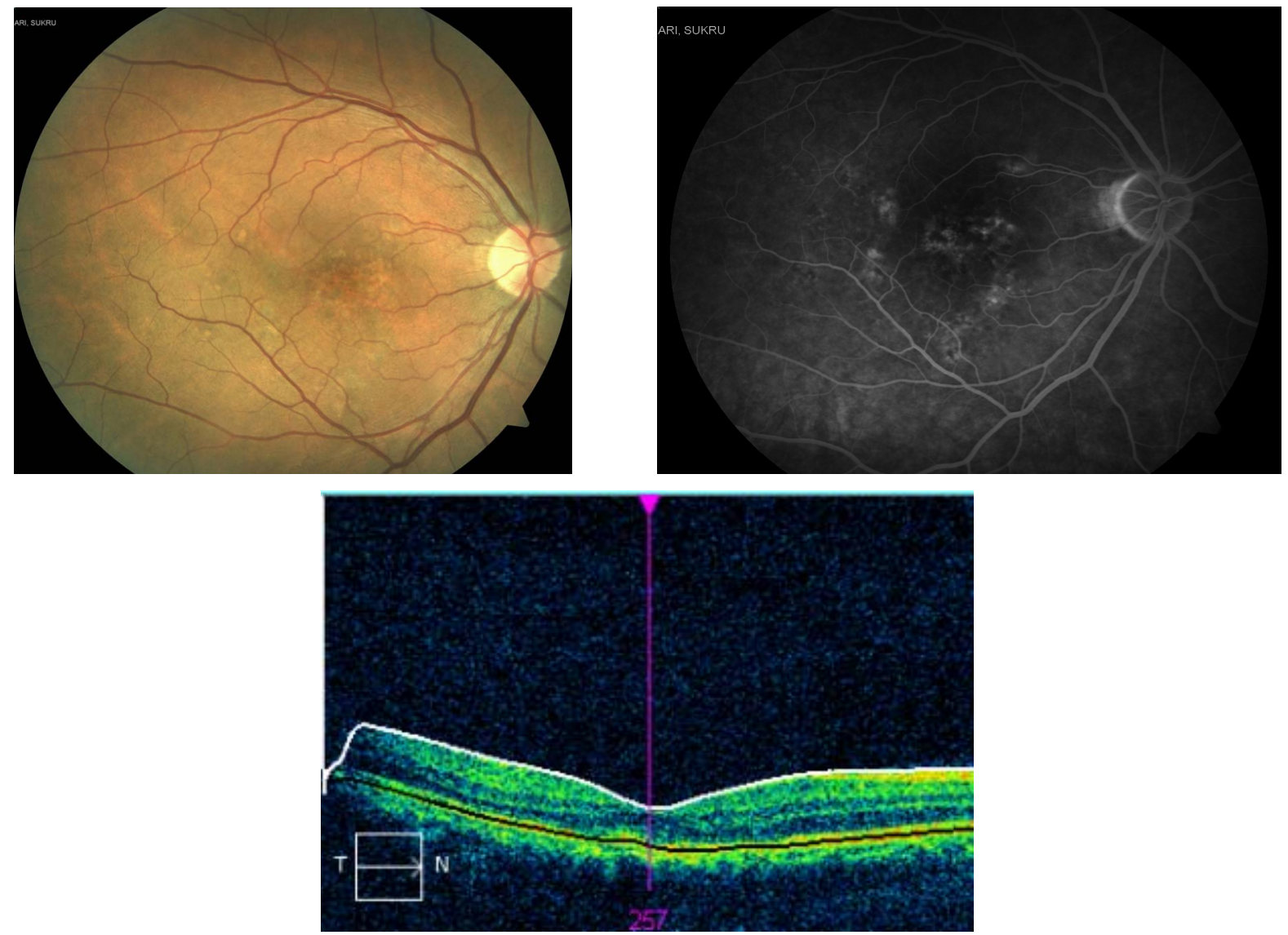

Figure 4. Clinical color photograph, flourescein angiography (late phases) and optical coherence tomography demonstreating complete resolution of CNV and subretinal fluid.

In our case, chronic CSR was succesfully treated with $2.5 \mathrm{mg}$ of single dose intravitreal bevacizumab injection. However CNV occured one year after the bevacizumab, then we decided to try repeat injection of another VEGF antibody ranibizumab for the management of CNV. Four week later, subretinal fluid and neovascular membrane was completely resolved and the vision improved to 20/20 and remained stable within one year. Both ranibizumab and bevacizumab, which are derived from the same parent molecule, inhibit all isoforms of VEGF. However, molecular and pharmacologic properties of these agents differ in several aspects. In an experimental animal model [10], ranibizumab has been shown to penetrate the choroid rapidly after an intravitreal injection. Bevacizumab is a three times larger molecule. Investigations, however, have proven the presence of bevacizumab throughout the neural retina, in the subretinal space and choriocapillaris within 24 hours of intravitreal injection.

Although CNV may complicate the natural history of CSR, in this case $2.5 \mathrm{mg}$ single dose of bevacizumab did not prevent the devoloping CNV secondary to CSR. In 2008, Wang et al. [11] reported that subretinal granular deposits from the phagocytosis photoreceptor segment, accumulating after retinal detachment could prevent the anti-VEGF treatment working in chronic CSR. Accordingly, Schaal et al. hypothesized that VEGF expression might be higher in patients with chronic CSR compared to patients with wet age-related macular degeneration (AMD) because affected areas are often multiple and widespread and not limited to the central part of the retina like in AMD and consequently might require higher doses of anti-VEGFs [12]. The same investigator reported that $50 \%$ of the cases demonstrated a complete resolution of subretinal fluid after treatment with $2.5 \mathrm{mg}$ bevacizumab [12].

More recently, Kaiser et al. [13], shown that ranibizumab with a fixed 12-month dosing regimen of $0.5 \mathrm{mg}$ has a favorable safety and efficacy profiles in patients with subfoveal CNV unresponsive to pegaptanib and bevacizumab. They explained this superiority with the fact that ranibizumab has a lower molecular weight and higher affinity to VEGF-A, which theoretically implies that it could better penetrate the retina and access the choroidal neovascular complex more readily. In addition, Rosenfeld et al. [14] reported that multiple intravitreal ranibizumab at escalating doses ranging from 0.3 to 2.0 
mg were well tolerated and biologically active in eyes with neovascular AMD within 5-months. Guided by this researches in this case we performed repeat intravitreal ranibizumab injection in CNV after bevacizumab injecttion for chronic CSR and it appeared to be an effective treatment option. Our experience in this case was unable to prove that bevacizumab $2.5 \mathrm{mg}$ could prevent the development of $\mathrm{CNV}$ as a complication of chronic CSC. Also in this case, ranibizumab was not found superior to bevacizumab in the treatment of the disease itself and in the prevention of its complications. However, we can hypothesize that a single dose of anti-VEGF may remain insufficient for improving the natural course of the disease and multiple doses may be more effective as in wet AMD. Further comparative studies with larger series and longer follow-up periods are needed in order to achieve a definitive conclusion on the role of anti-VEGF agents in the management of CSR.

\section{Acknowledgments}

The authors indicate no government or non-government financial support.

\section{REFERENCES}

[1] R. H. Loo, I. U. Scott, H. W. Flynn Jr., J. D. Gass, T. G. Murray, M. L. Lewis, P. J. Rosenfeld and W. E. Smiddy, "Factors Associated with Reduced Visual Acuity during Long-Term Follow-Up of Patients with İdiopathic Central Serous Chorioretinopathy," Retina, Vol. 22, No. 1, 2002, pp. 19-24 doi:10.1097/00006982-200202000-00004

[2] H. Matsunaga, K. Nangoh, M. Uyama, H. Nanbu, Y. Fujiseki and K. Takahashi, "Occurrence of Choroidal Neovascularization Following Photocoagulation Treatment for Central Serous Retinopathy," Nihon Ganka Gakkai Zasshi, Vol. 99, 1995, pp. 460-468.

[3] W. M. Chan, T. Y. Lai, D. T. Liu and D. S. Lam, "Intravitreal Bevacizumab (Avastin) for Choroidal Neovascularization Secondary to Central Serous Chorioretinopathy, Secondary to Punctate İnner Choroidopathy, or of İdiopathic Origin," American Journal of Ophthalmology, Vol. 143, No. 6, 2007, pp. 977-983. doi:10.1016/j.ajo.2007.02.039

[4] B. A. Cooper and M. A. Thomas, "Submacular Surgery to Remove Choroidal Neovascularization Associated with Central Serous Chorioretinopathy," American Journal of Ophthalmology, Vol. 130, No. 2, 2000, pp. 187-191 doi:10.1016/S0002-9394(00)00395-0

[5] A. Koytak, K. Erol, E. Coskun, N. Asik, H. Oztürk, Y. Ozertürk, "Fluorescein Angiography-Guided Photodynamic Therapy with Half-Dose Verteporfin for Chronic
Central Serous Chorioretinopathy," Retina, Vol. 30, No. 10, 2010, pp. 1698-1703. doi:10.1097/IAE.0b013e3181da4354

[6] J. F. Arevalo, R. A. Garcia-Amaris, J. A. Roca, et al., "Primary Intravitreal Bevacizumab for the Management of Pseudophakic Cystoid Macular Edema Pilot Study of the Pan-American Colloborative Retina Study Group," Journal of Cataract \& Refractive Surgery, Vol. 33, No. 12, 2007, pp. 2098-2105. doi:10.1016/j.jcrs.2007.07.046

[7] D. Iturralde, R. F. Spaide, C. B. Meyerle, J. M. Klancnik, L. A. Yannuzzi, Y. L. Fisher, J. Sorenson, J. S. Slakter, K. B. Freund, M. Cooney and H. F. Fine, "Intravitreal Bevacizumab (Avastin) Treatment of Macular Edema in Central Retinal Vein Occlusion: A Short-Term Study," Retina, Vol. 26, No. 3, 2006, pp. 279-284. doi:10.1097/00006982-200603000-00005

[8] D. Kook, A. Wolf, T. Kreutzer, A. Neubauer, R. Strauss, M. Ulbig, A. Kampik and C. Haritoglou, "Long-Term Effect of İntravitreal Bevacizumab (Avastin) in Patients with Chronic Diffuse Diabetic Macular Edema," Retina, Vol. 28, No. 8, 2008, pp. 1053-1060. doi:10.1097/IAE.0b013e318176de48

[9] S. J. Lim, M. I. Roh and O. W. Kwon, "Intravitreal Bevacizumab İjection for Central Serous Chorioretinopathy," Retina, Vol. 30, No. 1, 2010, pp. 100-106. doi:10.1097/IAE.0b013e3181bcf0b4

[10] J. Gaudreault, D. Fei, J. C. Beyer, A. Ryan, L. Rangell, V. Shiu, L. A. Damico, "Pharmacokinetics and Retinal Distribution of Ranibizumab, a Humanized Antibody Fragment Directed against VEGF-A, Following Intravitreal Administration in Rabbits," Retina, Vol. 27, No. 9, 2007, pp. 1260-1266. doi:10.1097/IAE.0b013e318134eecd

[11] M. Wang, I. C. Munch, P. W. Hasler, C. Prünte and M. Larsen, "Central Serous Chorioretinopathy," Acta Ophthalmologica, Vol. 86, No. 2, 2008, pp. 126-145. doi:10.1111/j.1600-0420.2007.00889.x

[12] K. B. Schaal, A. E. Hoeh, A. Scheuerle, F. Schuett and S. Dithmar, "Intravitreal Bevacizumab for Treatment of Chronic Central Serous Chorioretinopathy," European Journal of Ophthalmology, Vol. 19, No. 4, 2009, pp. 613617.

[13] R. S, Kaiser, O. P. Gupta, C. D. Regillo, A. C. Ho, M. S. Fineman, J. F. Vander, J. A. McNamara and G. C. Brown, "Ranibizumab for Eyes Previously Treated with Pegaptanib or Bevacizumab without Clinical Response," Ophthalmic Surgery, Lasers and Imaging, Vol. 43, No. 1, 2012, pp. 13-19.

[14] P. J. Rosenfeld, J. S. Heier, G. Hantsbarger and N. Shams, "Tolerability and Efficacy of Multiple Escalating Doses of Ranibizumab (Lucentis) for Neovascular Age-Related Macular Degeneration," Ophthalmology, Vol. 113, No. 4, 2006, pp. 623-632. doi:10.1016/j.ophtha.2006.01.027 